\title{
Dislocation BASED Fracture MeChaNICS
}


This page is intentionally left blank 

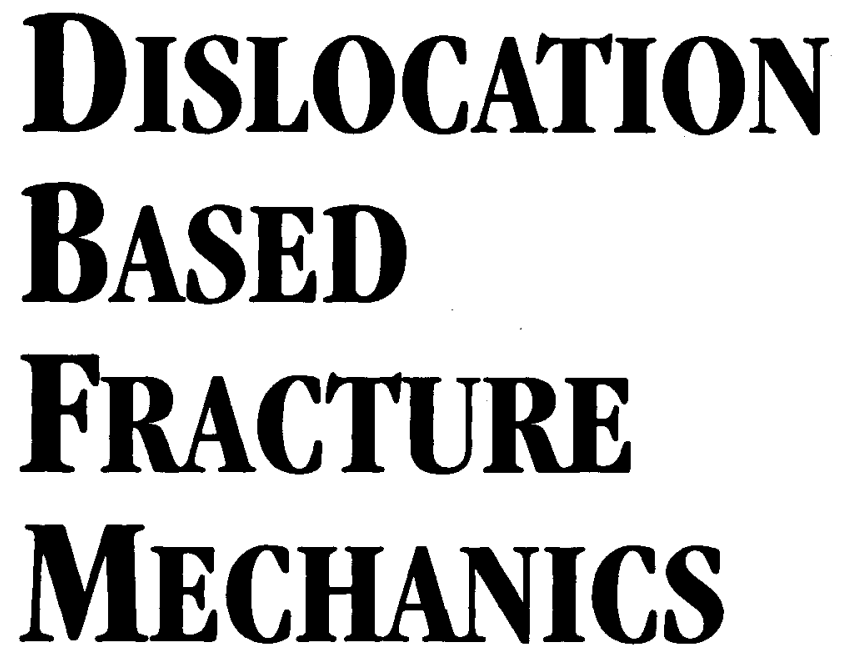

\section{Johannes Weertman}

Materials Science \& Engineering Department

Geological Sciences Department

Northwestern University 


\title{
Published by
}

World Scientific Publishing Co. Pte. Ltd.

5 Toh Tuck Link, Singapore 596224

USA office: 27 Warren Street, Suite 401-402, Hackensack, NJ 07601

UK office: 57 Shelton Street, Covent Garden, London WC2H 9HE

\author{
Library of Congress Cataloging-in-Publication Data \\ Weertman, Johannes. \\ Dislocation based fracture mechanics / Johannes Weetman. \\ p. $\mathbf{c m}$. \\ Includes bibliographical references and index. \\ ISBN-13 978-981-02-2620-6 \\ ISBN-10 981-02-2620-9
}

1. Fracture mechanics. 2. Dislocations in crystals. I. Title.

TA409.W44 1996

620.1'126--dc20

\section{5-51485}

CIP

\section{British Library Cataloguing-in-Publication Data \\ A catalogue record for this book is available from the British Library.}

First published 1996

Reprinted 1998, 2008

Copyright 1996 by World Scientific Publishing Co. Pte. Ltd.

All rights reserved. This book, or parts thereof, may not be reproduced in any form or by any means, electronic or mechanical, including photocopying, recording or any information storage and retrieval system now known or to be invented, without written permission from the Publisher.

For photocopying of material in this volume, please pay a copying fee through the Copyright Clearance Center, Inc., 222 Rosewood Drive, Danvers, MA 01923, USA. In this case permission to photocopy is not required from the publisher.

Printed in Singapore by B \& JO Enterprise 
To:

my wife

our children

our grandchildren

my parents

my brother
Julia

Julia and Bruce

Johannes and Willem

and their mother Leslie

Roelof and Christina

Willem and his wife Eleanor 


$$
\begin{gathered}
\text { The Magic Lengths of } \\
\text { Fracture Mechanics } \\
\frac{K^{2}}{\pi \sigma_{A}^{2}} \rightarrow \text { crack half length } \\
\frac{K^{2}}{\sigma_{y}^{2}} \rightarrow \approx \text { plastic zone size } \\
\frac{K^{2}}{G \sigma_{y}} \rightarrow \approx \text { crack opening displacement where plastic zone boundary crosses } \\
\text { crack plane behind tip }
\end{gathered}
$$

\section{Mnemonics}

$\mathscr{G}$ (energy release rate): $\quad$ George Irwin \& A. A. Griffith

$J$ Integral: Iock Eshelby \& Iames Rice

K (stress intensity factor): George Irwin \& Joseph $\underline{\text { Kies }}$ 


\section{PREFACE}

A crack in an elastic solid can be considered to be and, indeed, actually is a distribution of dislocations on the crack plane. A crack in an elastic-plastic solid not only is a distribution of dislocations on the crack plane but, in addition, is a distribution of non-redundant dislocations within the plastic zones of the crack tips. The stress-strain-rotation field of a discrete dislocation of unit strength is the Green's function from which the stress and elastic strain-rotation fields of a crack can be obtained. Moreover, the plastic strain-rotation field can be found from the motion and density of the dislocations in the crack tip plastic zones. To ignore the dislocation origin of the large stresses near a crack tip when attempting to find a crack solution is like attempting to solve a solid state physics problem oblivious to the fact that electrical fields arise from electrical charges.

Because the dislocation is the basic building block of the crack, fracture mechanics logically can be developed from its dislocation base. In dislocation texts it is noted that cracks in elastic solids can be modeled with dislocations ${ }^{\dagger}$. Texts on fracture mechanics, however, largely ignore the dislocation foundation of the crack. (Lardner's book is an exception. Lardner's book was written well before the current interest and recent research carried out on dislocation crack tip shielding and before any solution was found for a crack in an elastic-plastic solid using dislocation density fields.) In no dislocation text or fracture text is it noted that the problem of a crack in an elastic-plastic solid can be solved with use of dislocations. This text is the only one to present solutions for cracks in elasticplastic solids that are based on dislocation density fields. The dislocation density fields are obtained with the aid of the fundamental 'Maxwell's equations for dislocations' presented in Chapter 2. (These 'Maxwell dislocation equations' allow for the existence of a curiosity: a 'point' dislocation complex, or, conversely, the existence of an electron that is dislocation complex.)

I believe that any student entering the field of fracture will benefit from a text in which fracture mechanics is developed from its dislocation base. This book is my effort to supply such a text for the new graduate student or the investigator who is just entering the field. Portions of this book were used in advanced fracture courses offered at Northwestern University. I hope that the student studying this book can develop a dislocation 'world view' of cracks from which to see solutions of crack problems. The chapter on dislocations should give the student more than adequate background for understanding the application of dislocations to crack problems.

When I started to write this text only the problem of the stationary mode III crack in an elastic-plastic solid had been solved completely (without use of

† See the texts of Friedel (1964), Hirth and Lothe (1982) and Nabarro (1967). Apparently, the first dislocation based solution for the problem of the crack in an elastic solid is that obtained by Leibfreid. His solution employes a planar distribution of infinitesimal dislocations and the Hilbert transforms (Leibfried 1951). 
dislocations). No dislocation-based solution existed for any type of crack in an elastic-plastic solid. I put off completion of this book for some years until I found dislocation-based solutions for the three crack types in various elastic-plastic solids ${ }^{\ddagger}$. That is, for the crack opened in tension (mode I crack), the crack sheared in the direction perpendicular to the crack front (mode II crack), and the crack sheared in the direction parallel to the crack front (mode III crack). These dislocation-based solutions are the subjects of three chapters. It is evident from these solutions that complete, exact solutions for mode I and mode II cracks in general elastic-plastic solids are not likely to be found without explicit introduction of dislocations into the solution.

Any crack problem in an elastic solid can be solved starting from the stress field of a discrete dislocation. The stress field of dislocations distributed on a plane leads directly to the Hilbert transforms ${ }^{\dagger}$ and to the Muskhelishvili equations that are the tools to obtain crack solutions in elastic solids. A chapter in the book develops in detail how the Hilbert transforms and the Muskhelishvili equations are derived for the student who wants his understanding developed ab initio. Only the results of this chapter, not its derivations, are needed in the other chapters. The solution of the Bilby-Cottrell-Swinden-Dugdale crack, which is the simplest dislocation-based model crack with a (one dimensional) plastic zone, is easily obtained from the Muskhelishvili equations. Moreover, with these equations, as shown in Chapter 5 , the amount of dislocation crack tip shielding or antishielding is found in a straightforward way. At this point in the book all the dislocation tools are in place to find solutions for cracks in elastic-plastic solids. The following chapters present all the published and unpublished dislocation-generated solutions for cracks in elastic-plastic solids. These solutions are of infinitely long cracks in an isotropic solid.

The last section of the last chapter of the book discusses one paradox whose existence is only revealed in a dislocation approach to fracture. This puzzle is: the non-redundant dislocations are more numerous than redundant dislocations close to a crack tip if elastic-plastic stress-strain curves obtained under uniform strain conditions are valid under non-uniform strain conditions. But the redundant dislocation density is expected to be always greater than the non-redundant dislocation density. For experimentalists this discrepancy implies that they should provide theorists not only stress-strain curves obtained on uniformly deformed specimens but they should also give them information how stress-strain curves

F Exact analytical solutions were found for all three types of stationary cracks in a linear elastic-plastic solid and for the mode III crack in a work hardening solid and in an elastic perfectly plastic solid. Approximate analytical solutions were obtained for the growing mode III crack and for the stationary mode I crack and for the mode II crack in an elastic perfectly plastic solid.

${ }^{\dagger}$ Appendix $\mathrm{A}$ at the end of the book is a short table of Hilbert transforms. Appendix B is a long table of integrals that arise in crack problems but are not found in the usual integral tables. 
change if the plastic deformation is spatially very nonuniform. For theorists this paradox can be the starting point for predicting critical stress intensity factors from experimental stress-strain curve data. Until the redundant and nonredundant dislocation paradox is resolved there is little point in attempting to obtain corrections to any near crack tip stress-strain field solution that take into account the fact that strains are large in this region. Any proposed large strain constitutive equation used near a crack tip must lead to the result that the nonredundant dislocation density is greater than the redundant dislocation density. (It should be noted that in this book the derivations of dislocation density fields from plastic strain contain the implicit assumption that strain is small. It is shown in the last section of the book ( $\$ 10.6$ of Chapter 10$)$ that the region around a crack tip in which the strain is large is very small compared with the plastic zone size.)

My own research in the field of fatigue cracks and fracture, upon which part of this text is based, was supported in years past by the National Science Foundation, the Office of Naval Research, the Department of Energy, and the Air Force Office of Scientific Research. I thank these agencies for their former support. I thank Professor James S. Koehler, the co-proposer of the Zener-StrohKoehler crack and my thesis advisor, for introducing me to the wonderful world of the dislocation. I first came across elastic-plastic crack problems through the speakers at the mechanics colloquia that Dr. George Irwin, the father of fracture mechanics, ran at the U.S. Naval Research Laboratory. I confess that I understood virtually nothing of what was discussed at those long ago seminars. I was introduced, also many years ago, to the Muskhelishvili analysis used in part of this book while attempting to solve earthquake fault slippage problems during a sabbatical leave at the California Institute of Technology. I am grateful to Professor Robert Sharp, then department chairman of the Geological Sciences Division, for the invitation to spend a period of time at Caltech. That short period was the starting point for all my dislocation-based theoretical fracture work.

Over the years, I have been very fortunate for my own research to have the benefit of the stimulation from my, and my wife's, colleagues, students, coauthors at Northwestern University, Los Alamos National Laboratory, U. S. Army Cold Regions and Research Laboratory (CRREL), Scott Polar Research Institute, U. S. Naval Research Laboratory (NRL) and Paul Scherrer Institute. I am particularly indebted to Morris Fine and to Jerome Cohen for my career at Northwestern University and to Oscar Marzke for my career at NRL; to Walter Green for many research summers at Los Alamos; and to Anthony Gow, Chester Langway, Hans Röthlisberger, Wilford Weeks for my glaciological education at CRREL (and its predecessor: the U. S. Army Snow Ice and Permafrost Research Establishment (SIPRE)). I thank Jan Achenbach, Norman R. Adsit, Sean Agnew, Carolyn R. Aita, George S. Ansell, Anthony C. Arko, Richard J. Arsenault, Fred Boehme, John Barker, Zdenek Bazant, James Bender, H. M. Berg, G. Edward Birchfield, John Blacic, John E. Bloom, Mitchell L. Brandt, John E. Breen, John Brittain, Neil R. Brown, Robert Brown, Larry H. Burck, Jose Cabanas, Kent R. Carson, Hector 
Calderon, Parker Calkin, Anthony Calomino, Carolyn Campbell, C. A. (Dino) Caracostas, Show-Peng Chang, Yen-Chieng Chen, Rong-Tsang Chen, Herbert Cheng, Sam Chikwembani, Tsu-Wei Chou, Scott Clugston, David M. Cole, James Conley, Rebecca Cortez, John R. Cotner, Terry Creasey, Ralph C. Daehn, T. Davidson, Brijnandan Dehiya, Daniel P. Deroner, David Drewry, John Dundurs, Brian R. Elliott, Phillip Earvolino, Katherine Faber, John E. Flinn, Jr., Paul S. Follansbee, Kathryn Ann Forland, Gretchen Fougere, Earl Freise, Dershin Gan, Didier Gavillet, Prasad S. Godavarti, Dmitriy Gorelikov, Todd Gross, John E. Hack, Kishio Hidaka, R. Hanada, Rena L. Hecht, Sigfried S. Hecker, Randy Herrick, Jeffry Hillar, John Hillard, Peter Hobbs, John Hockett, Soon-Hyung Hong, John A. Horak, A. Van Hull, Thomas Isabell, Peter Jemian, Hitoshi Ishii, Lynette Angers Karabin, Jack Kauffman, Chul-Jung Kim, Sang-Tae Kim, Brad Kirkwood, Takanori Kiyohara, Luba Kmetyk, Cheryl Knepfler, Charles J. Kuehmann, In-Bae Kwon, S. K. Lahiri, Narin S. Lalwaney, Joseph Lane, Laura Lisiecki, Ing-Hour Lin, M. R. Lin, Tsu-An Lin, Henry Lippard, Guo-Zhi Lü, A. T. Lunde, David Luzzi, Kenneth D. Mahrer, R. H. Marion, Phillip N. LaMori, Harris L. Marcus, K. Maruyama, A. S. Mehner, Mike Meshii, Rahul Mitra, Jonathan S. Montgomery, Toshio Mura, Isabelle Muszynski, Sia Nemat-Nasser, Robert Nason, William Nieman, Gregory Olson, Richard Page, Y. K. Park, Donald Parkin, D. E. Pease, E. S. Pundarika, Arthur H. Purcell, V. R. (Sivan) Parameswaran, Hilda Richardson, Mindy Rittner, Timothy A. Roberts, Gordon Robin, Edward I. Salkovitz, Paul Sanders, Albert I. Schindler, Lyle Schwartz, Paul Shaninian, Adam L. Shepela, Hideki Shirai, H. M. Shodja, Howard W. Sizek, Walter F. Sommer, D. T. Spreng, Charles Swinthinbank, Sang-Hee Suh, J. C. Swartz, W. J. McG. (Greg) Tegart, George A. Themelis, R. Thom, Robb Thomson, Koichi Tsuchiya, Namio Urabe, J. B. VanderSande, Max Victoria, Jim Waber, Thomas B. Wade, Myang Yang, Don Whitmore, F. W. Wiffen, Andre Wilford, D. R. Williams, Ronald A. Yeske, Carl Youngdahl, Michael Zedalis, Eugene Zukas.

I thank Professor John Nye of the University of Bristol for submitting my first published paper on a dislocation-based plastic zone. An improved version of that analysis, on the growing mode III crack, is given in Chapter 6. Professor J. G. Dash of the University of Washington kindly suggested the statement in Chapter 2 on electric charge separation in thunderstorms. Members of my classes in which parts of this text was used pointed out misprints. Mr. Juan A. Hurtado of Northwestern University checked much of the material in this book. I also am indebted to him for use of a number of unpublished relationships that he derived, which are noted and acknowledged in the text. 


\section{TABLE OF CONTENTS}

\section{Preface}

vii

Selected Symbols List

xxii

\section{CHAPTER 1}

\section{Griffith-Inglis Crack \& Zener-Stroh-Koehler Crack}

1.1. Griffith-Inglis Crack

1.2. Stress Fields of Griffith-Inglis Cracks

1.2.1. Mode I Stress Field 6

1.2.2. Mode II Stress Field

1.2.3. Mode III Stress Field 14

1.2.4. Mode I Near Tip Stress Field 14

1.2.5. Mode II Near Tip Stress Field 16

1.2.6. Mode III Near Tip Stress Field 16

1.3. Elastic Displacement Fields of Griffith-Inglis Cracks 17

1.3.1. Mode I Near Tip Elastic Displacement Field 18

1.3.2. Mode II Near Tip Elastic Displacement Field 18

1.3.3. Mode III Near Tip Elastic Displacement Field 18

1.4. Fracture Stress of Griffith-Inglis Crack $\quad 18$

1.4.1. Energy Method 19

1.4.2. Critical Crack Tip Displacement Method 22

1.4.3. Dislocation Method 23

1.4.4. Stress Intensity Factor $\quad 24$

1.4.5. J Integral 25

1.4.6. Short Griffith-Inglis Crack 26

1.5. Zener-Stroh-Koehler Crack 27

1.6. Stress Fields of Zener-Stroh-Koehler Cracks 29

1.6.1. Mode I Near Tip Stress Field 30

1.6.2. Mode II Near Tip Stress Field 34

1.6.3. Mode III Near Tip Stress Field 35

1.7. Elastic Displacement Fields of Zener-Stroh-Koehler Cracks 35

1.7.1. Mode I Near Tip Elastic Displacement Field 36

1.7.2. Mode II Near Tip Elastic Displacement Field 36

1.7.3. Mode III Near Tip Elastic Displacement Field 36

1.8. Equilibrium Length of Zener-Stroh-Koehler Crack 37

1.8.1. Short Zener-Stroh-Koehler Crack 38

1.9. Equilibrium Length: ZSK Crack Subjected to an Applied Load 38

1.10. Energy of ZSK Crack Under an Applied Load 39

Chapter 1 Homework $\quad 42$ 


\section{CHAPTER 2}

Dislocation Mechanics $\quad 45$

2.1. Dislocation Stress Fields: Rectangular Coordinates 45

2.1.1. Glide Edge Dislocation 45

2.1.2. Climb Edge Dislocation 46

2.1.3. Screw Dislocation $\quad 47$

2.1.4. Elastic Strain and Rotation Fields $\quad 48$

2.2. Dislocation Stress Fields: Cylindrical Coordinates $\quad 48$

2.2.1. Glide Edge Dislocation $\quad 48$

2.2.2. Climb Edge Dislocation $\quad 49$

2.2.3. Screw Dislocation $\quad 49$

2.3. Crack Plane Traction and Non-Traction Stress 49

2.3.1. Crack Plane Traction Arising from a Crack Plane Dislocation Distribution

2.3.2. Crack Plane Non-Traction Arising from a Crack Plane

Dislocation Distribution $\quad 50$

2.4. Force on a Dislocation $\quad 53$

2.4.1. Extrinsic Resistance (Friction) Stress $\tau_{\mathrm{F}}$ to Dislocation Motion 54

2.4.2. Intrinsic Resistance (Friction) Stress $\tau_{\mathrm{i}}$ to Dislocation Motion 55

2.5. Dislocations and the $J$ Integral 55

2.6. Dislocation Crack Extension and Deflection Forces 58

2.6.1. Dislocation Crack Extension Force 58

2.6.2. Dislocation Crack Deflection Force 61

2.7. Dislocations Density Fields of Non-Redundant Dislocations 65

2.7.1. Antiplane Strain $\quad 66$

2.7.2. Plane Strain 67

2.7.3. Dislocation Density from Plastic Strain $\quad 70$

$\begin{array}{ll}\text { 2.7.3.1. Antiplane Strain } & 70\end{array}$

2.7.3.2. Antiplane Strain, Curvilinear Coordinates $\quad 71$

$\begin{array}{ll}\text { 2.7.3.3. Plane Strain } & 74\end{array}$

2.7.3.4. Plane Strain, Curvilinear Coordinates $\quad 78$

2.7.3.5. Incremental Form $\quad 80$

2.7.3.6. Strain Gradient Form Dislocation Density Field in Plane Strain 81

2.8. Electrical Analogy $\quad 81$

2.8.1. Dislocation Analogue of Point Electrical Charge $\quad 84$

2.9. Dislocations, the Source of Internal Stresses 86

2.9.1. General Dislocation Density Field 87

2.9.2. Lattice Curvature $\quad 89$

2.10. Edge Dislocation Stress-Strain-Rotation Field in Plane Stress $\quad 89$

2.10.1. Glide Edge Dislocation $\quad 90$ 
2.10.2. Climb Edge Dislocation 90

2.10.3. Mode I Crack and Mode II Crack in an Elastic Foil 91

2.10.4. Dislocation Crack Extension Force of Fracture Stress in Plane Stress 92

2.10.5. Screw Dislocation 92

2.11. Dislocations Near and on an Interface 93

2.11.1. Screw Dislocation 93

2.11.2. Glide Edge Dislocation 93

2.11.3. Climb Edge Dislocation 99

2.11.4. Comninou-Dundurs Equation 94

Chapter 2 Appendix: Non-Traction Stress Jump Without(?) a Planar Dislocation Distribution $\quad 96$

Test of the Rule $\quad 99$

Chapter 2 Homework 99

CHAPTER 3

Hilbert Transforms \& Muskhelishvili Equations 103

3.1. Hilbert Transforms 103

3.2. Muskhelishvili Equations 106

3.2.1. Determination of Muskhelishvili Equations for a Single Zone $\quad 107$

3.3. Determination of the Distance $c$ to the Outer Crack Boundary 110

3.4. Summary of Equations for a Single Dislocation Zone 113

3.5. Solution When the Stress is Infinite at the Crack Tips 114

$\begin{array}{ll}\text { 3.6. Non-Centered Coordinate System } & 116\end{array}$

3.7. Multiple Dislocation Zones $\quad 117$

3.7.1. Double Zone 117

3.7.2. Infinite Crack Tip Stress for Symmetric Double Zone 121

3.7.3. Symmetric Triple Zone $\quad 122$

3.8. Plane Stress 123

3.9. Resolution of a Hilbert Transform Paradox with
Ghost Dislocation Distributions

Table $3.1 \quad$. 127

$\begin{array}{ll}\text { Chapter } 3 \text { Homework } & 131\end{array}$

CHAPTER 4

Bilby-Cottrell-Swinden-Dugdale (BCSD) Crack 133

4.1. Bilby-Cottrell-Swinden-Dugdale (BCSD) 133

4.1.1. Griffith-Inglis Crack Limit 137

4.1.2. Crack Extension force of BCSD Crack 137

4.1.3. Crack Tip Shielding of BCSD Crack 138 
4.1.4. Mixed Mode I \& II BCSD Crack

4.1.4.1. Crack Extension Force

4.1.4.2. Crack Deflection Force

138

4.2. BCSD Type Zener-Stroh-Koehler Crack 139

4.2.1. Classical Limit of Zener Stroh-Koehler Crack

4.2.2. Stressed Zener-Stroh-Koehler Crack 142

4.3. Stress Fields of BCSD Cracks $\quad 143$

4.3.1. Mode III BCSD Crack 144

4.3.2. Mode II BCSD Crack 146

4.3.3. Mode I BCSD Crack 147

4.4. Asymmetric Crack Solutions 147

4.5. Double Slip Plane Crack Model 150

4.S.1. Stationary Crack 151

4.5.2. Crack Tip Stress Intensity Factor 154

4.5.2.1. Short Short-Crack 156

4.5.2.2. Dislocation Crack Tip Shielding and Antishielding 157

4.5.3. Growing Crack 158

4.5.3.1. R-Curve 159

Chapter 4 Homework $\quad 160$

\section{CHAPTER 5}

Crack Tip Shielding and Antishielding by Dislocations 163

5.1. Dislocation Crack Tip Shielding and Antishielding 165

5.1.1. Screw Dislocations 166

5.1.2. Glide Edge Dislocations 167

5.1.3. Climb Edge Dislocations 167

5.1.4. Stress Intensity Factor L for an Individual Dislocation 168

5.1.5. Near Tip $\quad 168$

5.1.5.1. Screw Dislocation 168

5.1.5.2. Glide Edge Dislocation 168

5.1.5.3. Climb Edge Dislocation $\quad 168$

$\begin{array}{ll}\text { 5.1.6. Far Crack } & 169\end{array}$

5.1.6.1. Screw Dislocation 169

5.1.6.2. Glide Edge Dislocation 169

5.1.6.3. Climb Edge Dislocation $\quad 169$

5.1.7. Near Tip Radial Edge Dislocation $\quad 170$

5.1.8. Near Tip Azimuthal Edge Dislocation 170

5.1.9. Comparison of $L$ with Stress Fields of Griffith-Inglis and
Zener-Strob-Koebler Cracks

5.1.10. Placing a Discrete Dislocation within the Crack 174

5.2. Induced Crack Plane Dislocation Distribution $B(x) \quad 174$

5.2.1. Screw Dislocation Induced 174 
5.2.2. Glide Edge Dislocation Induced 176

5.2.3. Climb Edge Dislocation Induced 177

5.2.4. Near Tip 177

5.2.4.1. Screw Dislocation Induced 177

5.2.4.2. Glide Edge Dislocation Induced 178

5.2.4.3. Climb Edge Dislocation Induced 178

5.2.5. Near Tip Radial Edge Dislocation Induced 178

5.2.6. Near Tip Azimuthal Edge Dislocation Induced 179

5.3. Stress on Crack Plane Ahead of Crack Tip 179

5.3.1. Screw Dislocation 180

5.3.2. Glide Edge Dislocation $\quad 180$

5.3.3. Climb Edge Dislocation $\quad 180$

5.3.4. Near Tip 181

5.3.5. Near Tip Crack Plane Dislocation arising from Near Tip Dislocation 182

5.4. Impurity Atom Crack Tip Shielding and Antishielding 184

5.4.1. Near Tip 185

5.4.2. Far Crack 186

5.5. Microcrack Shielding and Antishielding 187

5.5.1. Dislocation Dipoles 189

5.5.1.1. Horizontal Glide Edge Dislocation Dipole $\quad 189$

5.5.1.2. Vertical Glide Edge Dislocation Dipole 190

5.5.1.3. Horizontal Climb Edge Dislocation Dipole 190

5.5.1.4. Vertical Climb Edge Dislocation Dipole 191

5.5.1.5. Horizontal Screw Dislocation Dipole 191

5.5.1.6. Vertical Screw Dislocation Dipole 191

5.5.2. Near Tip Stress Intensity Factors 191

5.5.2.1. Horizontal Glide Edge Dislocation Dipole 192

5.5.2.2. Vertical Glide Edge Dislocation Dipole 192

5.5.2.3. Horizontal Climb Edge Dislocation Dipole 192

5.5.2.4. Vertical Climb Edge Dislocation Dipole 192

5.5.2.5. Horizontal Screw Dislocation Dipole 192

5.5.2.6. Vertical Screw Dislocation Dipole 193

5.6. Crack Tip Blunting by Dislocation Emission 193

5.6.1. Rice-Thomson Model 193

5.6.2. Model Based on Muskhelishvili Type Analysis 195

5.6.3. Crack Tip Blunting 198

Chapter 5 Homework 199

CHAPTER 6

Mode III Crack in an Elastic-Plastic Solid 201

6.1. Mode III Crack in an Elastic Perfectly Plastic Solid 201

6.1.1. Stress Field $\quad 202$ 
6.1.2. Strain Field 206

6.1.3. Dislocation Crack Extension Force and the Plastic Zone Size 207

6.1.4. Crack Tip Shielding 207

6.1.5. Crack Plane Dislocation Distribution 208

6.1.6. Elastic Region Stress Field 208

6.1.7. Boundary Conditions Summary 210

6.2. Stationary Crack in a Work Hardening Solid in Small Scale Yielding 211

6.2.1. Stress Solution $\quad 212$

6.2.2. Strain Solution $\quad 214$

6.2.3. Dislocation Density Field 214

6.2.4. Slip Trajectories $\quad 214$

6.2.5. Plastic Strain from Dislocation Density Field 217

6.2.6. Dislocation Crack Tip Shielding 220

6.2.7. Dislocation Crack Extension Force 220

6.2.8. Crack Plane Dislocation Density Distribution 221

6.2.9. Net Burgers Vector of Plastic Zone Dislocations 222

6.3. Mode III Crack in a Linear Work Hardening Solid with Zero Yield Stress 222

6.3.1. Stress Solution 223

6.3.2. Maximum Shear Stress Plane Trajectories 224

6.3.3. Dislocation Density 225

6.4. Mode III Crack in an Elastic Perfectly Plastic Solid
in Large Scale Yielding

$\begin{array}{ll}\text { in Large Scale Yielding } & 226 \\ \text { 6.4.1. Approximate Solution } & 227\end{array}$

6.4.2. Special Problem 228

6.4.3. Exact Solution $\quad 230$

6.4.3.1. 'Pressurized' Mode III Crack 235

6.5. Growing Mode III Crack in an Elastic Perfectly Plastic Solid 236

6.5.1. Chitaley-McClintock Asymptotic Region 238

6.5.2. Strain Field in CM Sector $\quad 239$

6.5.3. Strain Field in DH Sector $\quad 242$

6.5.4. Approximate Solution 243

Chapter 6 Appendix: Iterative Solution of Special Problem 249

Chapter 6 Homework $\quad 256$

\section{CHAPTER 7}

Mode II Crack in an Elastic-Plastic Solid

7.1. Mode II Crack in an Elastic Perfectly Plastic Solid in Small Scale Yielding 259

7.1.1. Plastic Zone Stress Field

7.1.2. Asymptotic Dislocation Density Field

7.1.2.1. Asymptotic Dislocation Density Field from Stress Field 265

7.1.2.2. Asymptotic Dislocation Density Field from Strain Field 265

7.1.3. Plastic Zone Strain-Rotation Field and Dislocation Density Field 266 
7.1.4.1. Dislocation Crack Extension Force and the Plastic Zone Size 270

7.1.4.2. Dislocation Crack Tip Shielding $\quad 270$

7.1.5. Crack Plane Dislocation Distribution 273

7.1.6. Elastic Region Stress Field 275

7.1.6.1. Constant Stress Magnitude Contours 279

7.2. Mode II Crack in a Linear Work Hardening Solid with Zero Yield Stress 282

7.2.1. Stress Solution 283

7.2.2. Maximum Shear Stress Plane Trajectories 284

$\begin{array}{ll}\text { 7.2.3. Rotation } & 285\end{array}$

7.2.3.1. A Physical Inconsistency 285

7.2.4. Solution without Reversed Slip $\quad 286$

7.2.4.1. Dislocation Density Field 286

$\begin{array}{ll}\text { Chapter } 7 \text { Homework } & 289\end{array}$

\section{CHAPTER 8}

Mode I Crack in an Elastic-Plastic Solid 291

8.1. Mode I Crack in an Elastic Perfectly Plastic Solid in Small Scale Yielding 291

8.1.1. Mode II Analogue Plastic Zone Stress Field 292

8.1.2. Asymptotic Dislocation Density Field 293

8.1.3. Plastic Zone Stress Field: Mode I Crack in an

Elastic Perfectly Plastic Solid Dislocation Density Field 299

8.2. Dislocation Density Field $\quad 304$

8.2.1. Strain-Rotation Field $\quad 306$

8.2.1.1. Stream Functions and Displacement Fields 306

8.2.1.2. Strain-Rotation Field 307

8.2.2. Dislocation Density Field 308

8.3. Dislocation Crack Extension Force 309

8.3.1. Transformed Dislocation Density Components $\quad 310$

$\begin{array}{ll}\text { 8.3.2. Transformed Stress Components } & 310\end{array}$

8.4. Dislocation Crack Tip Shielding $\quad 310$

8.4.1. Trajectory Equations 311

8.5. Crack Plane Dislocation Distribution 316

8.6. Elastic Region Stress Field 316

8.6.1. Constant Stress Magnitude Contours 319

8.7. Mode I Crack in a Linear Work Hardening Solid with Zero Yield Stress 322

8.7.1. Stress Solution 322

8.7.2. Maximum Shear Stress Plane Trajectories $\quad 323$

$\begin{array}{ll}\text { 8.7.3. Rotation } & 324\end{array}$

8.7.3.1. A Physical Inconsistency $\quad 324$

8.7.4. Solution without Reversed Slip $\quad 325$ 
8.7.4.1. Dislocation Density Field 325

8.7.5. Dislocation Density Field Found from Plastic Strain Field 327

8.8. Hutchinson-Rice-Rosengren (HRR) Stress-Strain Field 329

8.8.1. Boundary and Symmetry Conditions 330

8.8.2. HRR Stress Field $\quad 332$

8.8.3. HRR Strain Field 333

8.8.4. HRR Strain-Strain Field for a Special Case 334

8.8.5. Dislocation Density Field of the HRR Stress-Strain-Rotation Field 337

8.8.6. Dislocation Density Condition 338

8.8.7. Exponent Condition 339

8.9. Mixed Mode I \& Il Stationary Crack in an Elastic Perfectly Plastic Solid 342

8.9.1. Shih Asymptotic Stress Field 342

8.9.2. Asymptotic Stress Field with Sector Below Yield Stress 344

$\begin{array}{ll}\text { Chapter } 8 \text { Homework } & 348\end{array}$

\section{CHAPTER 9}

Moving Yoffe Crack

9.1. Dislocation Stress Fields 351

9.1.1. Uniformly Moving Screw Dislocation Stress Field 352

9.1.2. Uniformly Moving Glide Edge Dislocation Stress Field 352

9.1.3. Uniformly Moving Climb Edge Dislocation Stress Field 353

9.1.4. Dislocation and Crack Plane Traction Stresses 354

9.2. Stress Fields of Moving Yoffe Cracks $\quad 356$

9.2.1. Mode I Crack 357

9.2.2. Mode II Crack 357

$\begin{array}{ll}\text { 9.2.3. Mode III Crack } & 358\end{array}$

9.2.4. Mode I Near Tip Stress Field 358

9.2.5. Mode II Near Tip Stress Field 359

9.2.6. Mode III Near Tip Stress Field 359

9.2.7. Plane of Maximum Tensile Stress at Mode I Crack Tip 360

9.2.7.1. Yoffe Crack Branching $\quad 361$

9.2.8. Plane of Maximum Tensile and Shear Stress at Mode II Crack Tip 365

9.2.9. Plane of Maximum Shear Stress at Mode III Crack Tip 370

9.3. Dislocation Density Field Equations $\quad 370$

9.3.1. Antiplane Strain $\quad 371$

9.3.2. Plane Strain $\quad 372$

Chapter 9 Homework $\quad 372$

CHAPTER 10

Interesting Applications $\quad 373$

10.1. Crevasses and Dikes $\quad 373$ 
10.1.1. Crevasses

10.1.2. Propagation of Magma Filled Cracks 379

10.1.3. Dikes

10.2. Unstable Slippage

10.3. Edge Cracks in Elastic Solids $\quad 385$

10.4. Interface Cracks 391

10.5. Fatigue Crack Propagation 398

10.5.1. Fatigue Crack Growth Controlled by Crack Tip Blunting 398

10.5.2. Paris Law 399

10.6. Redundant and Non-Redundant Dislocations 402

10.6.1. Irwin-Orowan Fracture Equation $\quad 405$

Chapter 10 Appendix: Stress Field of a Mode I and of a Mode II Interface Crack $\quad 407$

Chapter 10 Homework 413

APPENDIX A

$\begin{array}{ll}\text { Short Table of Hilbert Transforms } & 417\end{array}$

\section{APPENDIX B}

Table of Useful Integrals for Crack Problems

\section{APPENDIX C}

Stress Fields of Dislocations Near or On an Interface 439

C.1. Screw Dislocation

C.1.1. Discrete Image Screw Dislocations 439

C.1.2. Image Screw Interface Dislocation Distributions 441

C.2. Glide Edge Dislocation $\quad 442$

C.2.1. Continuity Conditions 443

C.2.2. Real Dislocation and Set I of Image Dislocations 443

C.2.3. Set II of Image Dislocations $\quad 445$

C.2.4. Shear Stress Continuity $\quad 446$

C.2.5. Normal Stress Continuity $\quad 447$

C.2.6. Normal Displacement Continuity $\quad 448$

C.2.7. Tangential Displacement Continuity 449

$\begin{array}{ll}\text { C.2.8. Constants } & 450\end{array}$

C.3. Climb Edge Dislocation 451

C.4. Dislocation at the Interface 451

C.4.1. Screw Dislocation 451

C.4.2. Glide Edge Dislocation 451 
C.4.3. Climb Edge Dislocation

C.5. Comninou-Dundurs Equations

C.6. Inverse Comninou-Dundurs Equations

\section{APPENDIX D}

\section{Table of Useful Equations}

D.1. Stress and Strain Components

D.1.1. Cartesian Coordinates

D.1.2. Cylindrical Coordinates

D.1.3. Finger and Thumb Coordinates

D.1.4. Strain Compatibility Condition for Plane Strain

D.2. Elastic Constants

D.3. Stress and Strain Components in Rotated Coordinate System

D.4. Conditions for Antiplane Strain, Plane Strain and Plane Stress

D.5. Equations for Dynamics Equilibrium

D.6. Non-Redundant Dislocation Density Field

D.6.1. Cartesian Coordinates

D.6.1.1. Frank's Rule

D.6.2. Cylindrical Coordinates

D.6.2.1. Frank's Rule

D.6.3. Spherical Coordinates

D.6.3.1. Frank's Rule

D.6.4. Dislocation Density Relationships and Strain Compatibility Equations

D.6.4.1. Beltrami-Michell (Strain) Compatibility Equations

D.7. Stress-Strain-Rotation Displacement Fields in Plane Strain from Stress and Strain Functions

D.7.1. Airy Stress Function

D.7.2. Displacement and Rotation Field

D.7.3. Stream Function

D.7.4. Antiplane Strain Stress Function

D.8. The Del Divergence, Curl and Gradient Operators 467

D.8.1. Cartesian

D.8.2. Cylindrical

D.8.3. Spherical

D.8.4. Cylindrical, Shifting Center

D.9. Equations of Static Equilibrium in Shifting Center Cylindrical

Coordinate System 
D.10. Trigonometric Relationships 469

D.11. Dirac Delta Functions $\quad 470$

D.11.1. One Dimension $\quad 470$

D.11.2. Two Dimensions $\quad 470$

D.11.3. Three Dimensions $\quad 470$

D.11.4. One Dimension, Special Example 471

D.12. Curves 472

D.13. Surface Dislocation Density Tensor 472

D.14. Single Stress Component - Dislocation Density Relationships 474

D.14.1. Plane Strain $\quad 474$

D.14.2. Antiplane Strain $\quad 474$

APPENDIX E

Derivation of the Del Gradient and other Operators for the Shifting Center Cylindrical Coordinate System

APPENDIX F

Orthogonal Curvilinear Coordinates $\quad 481$

F.1. Curvilinear Coordinates $\quad 481$

F.1.1. Exponential Spirals $\quad 482$

F.1.2. Non-exponential Spiral $\quad 484$

F.2. Static Equilibrium Equations $\quad 487$

F.2.1. Plane Strain $\quad 487$

F.2.2. Antiplane Strain 493

F.3. Gradient $\nabla_{\text {grad }}$, Divergence $\nabla_{\text {div }}$ and Curl $\nabla_{\text {curl }}$ Operators 493

\section{APPENDIX G}

Dislocations in Stress Space

G.1. Cracks in Elastic Stress Space

G.1.1. Zener-Stroh-Koebler Crack 498

G.1.2. Griffith-Inglis Crack $\quad 501$

G.2. Plane Strain Stress Space 503

REFERENCES $\quad 505$

Dislocation Texts $\quad 505$

Fracture Texts $\quad 505$

$\begin{array}{ll}\text { Other Texts } & 507\end{array}$

Review Papers $\quad 508$

Research Papers $\quad 510$ 
xxii

\section{SELECTED SYMBOLS LIST}

$a$

$a_{\mathrm{R}}, a_{\mathrm{L}} \quad x=a_{\mathrm{R}}, x=-a_{\mathrm{L}}$ are positions of right and left hand crack tips when origin centered on plastic zone edges

$\mathrm{da} / \mathrm{dN} \quad$ growth rate per cycle of a fatigue crack

$\boldsymbol{b}$

$b$

$b_{\theta}$

$b_{\mathbf{r}}$

$b_{\mathrm{T}}$

$B(x)$

$\mathscr{B}$

$c$

$C_{\text {L }}$

$C_{\mathrm{R}}$

$\mathrm{C}_{\mathrm{S}}$

CTOD

$D(x)$

$F, T$

$F_{\mathrm{E}}$

$F_{\mathrm{D}}$

$\boldsymbol{g}$

G

$G^{*}$

$\overline{\mathrm{G}}$

crack length, one half of total length of internal crack (crack tips at $x= \pm a$ ) or total length of surface (edge) crack

Burgers vector of a discrete dislocation

length of Burgers vector of a discrete dislocation

azimuthal component of Burgers vector

radial component of Burgers vector

total Burgers vector of dislocations in a Zener-Stroh-Koehler crack

dislocation density of infinitesimal dislocations on a plane (in total Burgers vector per unit length);

dislocation density of infinitesimal dislocations

outer edge of plastic zones of internal crack are at $x= \pm c$

longitudinal wave velocity

Rayleigh wave velocity

shear wave velocity

crack tip opening displacement (at $x=a$ )

displacement across the crack plane. Equal to integral of $B(x)$.

subscripts for terms involved with finger and thumb trajectories

dislocation crack extension force

dislocation crack deflection force

gravitational acceleration

shear modulus (elastic)

shear modulus (linear plastic)

shear modulus (linear elastic-plastic) 
effective shear modulus $\bar{G}=2 G_{\mathrm{A}} G_{\mathrm{B}} /\left(G_{\mathrm{A}}+G_{\mathrm{B}}\right)$, interface crack Irwin's energy release rate

I, II, III subscripts for terms involved with modes I, II and III cracks

$\mathscr{I}, \mathscr{L}, \mathscr{K}, \mathscr{L}$ integrals for elastic displacements of Griffith-Inglis and ZenerStroh-Koehler cracks

'J' from J Integral

K

nominal stress intensity factor: For crack in infinite solid, $K=$ $\sigma_{\mathrm{A}}(\pi a)$

$K_{\mathrm{gc}}$

critical stress intensity factor for Griffith-Inglis crack for cleavage crack advance

$K_{\mathrm{c}}$

stress intensity factor at fracture

critical stress intensity factor for Griffith-Inglis crack for blunting

local stress intensity factor at crack tip

cyclic stress intensity factor: $\Delta K=\left(\sigma_{\max }-\sigma_{\min }\right) \sqrt{ }(\pi a)$

threshold cyclic stress intensity factor below which a long fatigue crack will not grow

$L$

dislocation (or impurity atom) shielding or antishielding stress intensity factor

n

unit normal to contour

PZS

plastic zone size

$\mathscr{R}_{\mathrm{F}}, \mathscr{R}_{\mathrm{T}}$

radius of curvature of finger and thumb trajectories

unit tangent vector

displacement vector (components $u, v$ and $w$ in the $x, y$ and $z$ directions or $u_{\mathrm{r}}, u_{\theta}$ and $w$ in the $r, \theta$ and $z$ directions)

V dislocation velocity or crack velocity

$\alpha$

$\alpha=(1-v)$ where $v$ is Poisson's ratio

$\alpha_{\mathrm{e}} \quad \alpha_{\mathrm{e}}=\alpha=(1-v)$

$\alpha_{\mathrm{i}} \quad \alpha_{\mathrm{i}}=\alpha_{\mathrm{e}}$ or $\alpha_{\mathrm{s}}$

$\alpha_{\mathrm{s}} \quad \alpha_{\mathrm{s}}=1$ 
$\tilde{\alpha} \quad \tilde{\alpha}=\frac{1}{2} \alpha=\frac{1}{2}(1-v)$ for mode II, crack $\tilde{\alpha}=1$ for mode III crack.

$\bar{\alpha} \quad \bar{\alpha}=(1+v)$ in plane stress problems

$\bar{\alpha} \quad \bar{\alpha}=$ effective alpha constant in interface crack problems

$\beta \quad$ Dundurs parameter, $\beta=$

$$
\frac{1}{2}\left[\left(1-2 v_{\mathrm{A}}\right) G_{\mathrm{B}}-\left(1-2 v_{\mathrm{B}}\right) G_{\mathrm{A}} y /\left[\alpha_{\mathrm{A}} G_{\mathrm{B}}+\alpha_{\mathrm{B}} G_{\mathrm{A}}\right]\right.
$$

$\beta_{\mathrm{L}} \quad \beta_{\mathrm{L}}=\left(1-\mathrm{V}^{2} / \mathrm{C}_{\mathrm{L}}{ }^{2}\right)^{1 / 2}$

$\beta_{S} \quad \beta_{S}=\left(1-V^{2} / C_{S}{ }^{2}\right)^{1 / 2}$

$\beta_{2 s} \quad \beta_{2 s}=\left(1-V^{2} / 2 C_{s}{ }^{2}\right)^{1 / 2}$

$\gamma \quad$ surface energy

$\epsilon_{\mathrm{ij}} \quad$ tensor strain components

$\epsilon, \epsilon^{c}, \epsilon^{p} \quad$ strain magnitude, elastic strain, plastic strain

$\lambda, \mu \quad$ Lamé elastic constants

Poisson's ratio

$\rho_{i} \quad$ density of either solid, liqiud, ice, water, etc.

$\rho_{N} \quad$ dislocation density of discrete non-redundant dislocations in plastic zone (in total number per unit area)

$\rho_{R} \quad$ dislocation density of discrete redundant dislocations in plastic zone (in total number per unit area)

$\sigma_{\mathrm{ij}} \quad$ tensor stress components

$\sigma_{i \omega} \quad \sigma_{i \omega} \equiv(G / \alpha)\left\{\omega_{i}\right\}_{\text {elastic }}=$ rotation pseudo-stress component

$\sigma \quad$ stress magnitude

$\sigma_{\mathrm{A}} \quad$ applied stress

$\omega, \omega^{\mathrm{e}}, \omega^{\mathrm{p}}$, rotation, elastic rotation, plastic rotation

$\omega_{\text {elastic }} \quad$ elastic rotation

$\omega_{\text {plastic }} \quad$ plastic rotation 